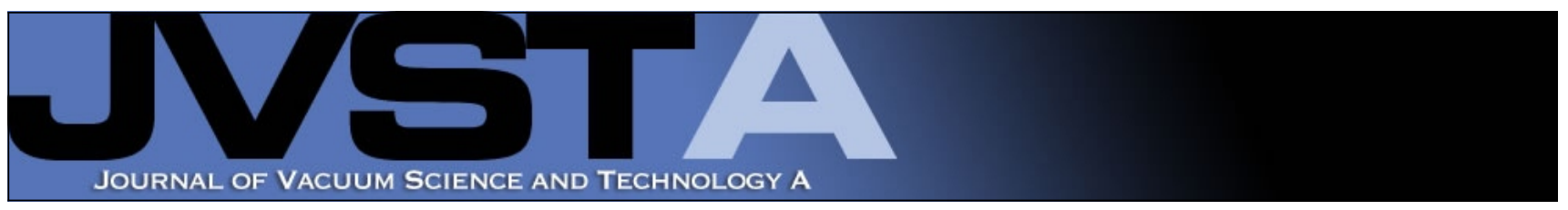

\title{
Monte Carlo modeling of electron beam physical vapor deposition of yttrium
}

Jing Fan, lain D. Boyd, and Chris Shelton

Citation: J. Vac. Sci. Technol. A 18, 2937 (2000); doi: 10.1116/1.1310656

View online: http://dx.doi.org/10.1116/1.1310656

View Table of Contents: http://avspublications.org/resource/1/JVTAD6/v18/i6

Published by the AVS: Science \& Technology of Materials, Interfaces, and Processing

\section{Additional information on J. Vac. Sci. Technol. A}

Journal Homepage: http://avspublications.org/jvsta

Journal Information: http://avspublications.org/jvsta/about/about_the_journal

Top downloads: http://avspublications.org/jvsta/top_20_most_downloaded

Information for Authors: http://avspublications.org/jvsta/authors/information_for_contributors

\section{ADVERTISEMENT}

\section{Instruments for advanced science}
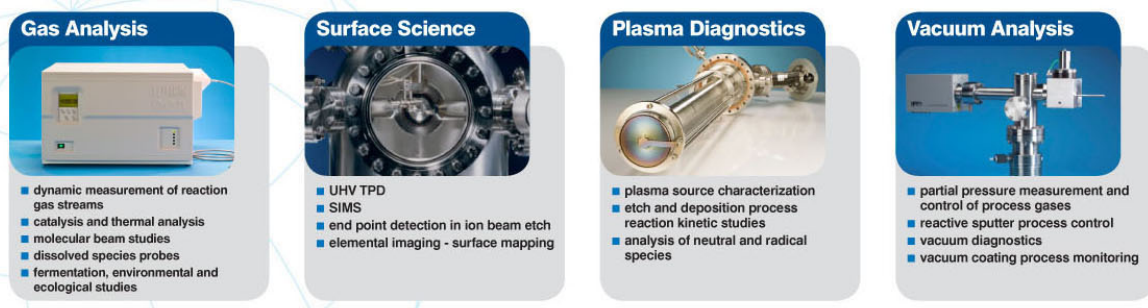

contact Hiden Analytical for further details

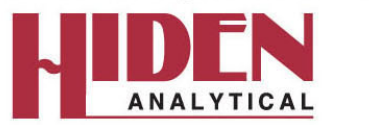

info@hideninc.com

www.HidenAnalytical.com

CLICK to view our product catalogue 


\title{
Monte Carlo modeling of electron beam physical vapor deposition of yttrium
}

\author{
Jing Fan ${ }^{\mathrm{a})}$ and lain D. Boyd \\ Department of Aerospace Engineering, University of Michigan, Ann Arbor, Michigan 48109 \\ Chris Shelton \\ Three M Company, St. Paul, Minnesota 55144
}

(Received 26 May 2000; accepted 24 July 2000)

\begin{abstract}
As part of a study on the deposition of superconducting films of $\mathrm{YBa}_{2} \mathrm{Cu}_{3} \mathrm{O}_{7-\delta}$, a three-dimensional electron beam physical vapor deposition process of yttrium in a vacuum chamber is investigated both computationally and experimentally. The numerical analysis employs the direct simulation Monte Carlo (DSMC) method. The experimental studies consist of atomic absorption spectra taken in the evaporated yttrium plume and deposited film thickness profiles. Some important modeling issues such as atomic collision cross sections for metal vapors and hyperfine electronic structure of the atomic absorption spectra are addressed. Film deposition thicknesses on the substrate and atomic absorption spectra given by the DSMC method and experiment are in excellent agreement. Collisions between the atoms are found to have a significant effect on the film growth rate and area of uniform deposition as the evaporation rate of yttrium increases. (C) 2000 American Vacuum Society. [S0734-2101(00)02206-5]
\end{abstract}

\section{INTRODUCTION}

Electron beam physical vapor deposition (EBPVD) is an important deposition technique for a variety of materials that involves the use of an electron beam to vaporize metallic atoms from a solid ingot. Applications of this technology include coating of high temperature superconducting films, metallic coating of fibers, and enrichment of uranium for nuclear power generation. A critical component of these processes is the manner in which the atoms are transported from the ingot to the deposition surface. There is a need to understand in detail the gas dynamics of the expansion process.

In this article, we consider yttrium vapor deposition both numerically and experimentally. This investigation is part of a study of the deposition of superconducting films of $\mathrm{YBa}_{2} \mathrm{Cu}_{3} \mathrm{O}_{7-\delta}$ that are of great technological interest in numerous applications. ${ }^{1}$ In the EBPVD process, atoms are vaporized from a liquid pool of an ingot of pure yttrium by a high-energy electron beam. The vapor jet expands through a chamber, impinges, and deposits on to a substrate. Using vacuum pumps, the background pressure is maintained at $2-5 \times 10^{-5}$ Torr. The vaporization rates achieved with the electron beam produce flow of relatively high density close to the ingot surface. However, as the jet proceeds toward the substrate, rapid expansion brings the flow into the noncontinuum regime. The focus of the present work was to provide a basis to understand and improve the EBPVD process carried out in the experimental facility.

The direct simulation Monte Carlo (DSMC) method $^{2}$ is used to simulate the three-dimensional gas dynamic process. It is a powerful and general numerical technique for computation of low density, nonequilibrium flows, and has been developed over the past 30 years primarily for aerospace

${ }^{\text {a)} E l e c t r o n i c ~ m a i l: ~ j i n g f a n @ e n g i n . u m i c h . e d u ~}$ applications. In the method, a relatively small number of model particles that is stored in a computer is used to represent the larger number of particles in real gas flows. The particles move through physical space and undergo collisions appropriate to the local flow conditions. Once the simulation has reached a steady state, macroscopic quantities such as density and temperature, as well as surface fluxes, are obtained by time averaging particle properties. The method provides detailed information on the gas flow at the level of particle velocity and energy distributions. A numerically efficient implementation of the DSMC method called MONACO has been developed and successfully verified for nonequilibrium flows. ${ }^{3,4}$

The application of the DSMC method to metal vapor flows is straightforward in principle and has been demonstrated in relatively simple geometries such as an axisymmetric case. ${ }^{5}$ Some specific complications do naturally arise. One of them is the determination of atomic collision cross sections for metal vapors, $\sigma_{T}$. Collisions between atoms change their trajectories that decide them moving toward the substrate or toward the chamber walls, and therefore significantly affect the film growth rate and area of uniform deposition that are key factors for film technology. Appropriate collision cross sections are essential for the DSMC method to simulate particle collisions. They are usually derived from coefficients of viscosity ${ }^{6}$ and have been obtained for many common gases such as nitrogen, argon, etc. ${ }^{2}$ Measurement of the transport properties for metal vapors at high temperature, however, has proven very difficult because of many unavoidable sources of error. ${ }^{7}$ Actually, except for alkali metal vapors, ${ }^{8-12}$ no viscosity data are available for metal vapors such as yttrium, barium, and copper, and few studies theoretically address the collision cross sections for metal vapors.

In the present article, we describe the development and assessment of a DSMC model for yttrium EBPVD. First, 
TABLE I. Interaction parameters for alkali metal vapors.

\begin{tabular}{cccc}
\hline \hline Element & $\eta$ & $\kappa$ & $A_{2}(\eta)$ \\
\hline Lithium & 6.333 & $7.984 E-70$ & 0.379 \\
Sodium & 7.351 & $4.979 E-79$ & 0.355 \\
Potassium & 6.737 & $4.462 E-73$ & 0.368 \\
Rubidium & 6.669 & $2.521 E-72$ & 0.370 \\
Cesium & 6.531 & $7.018 E-71$ & 0.374 \\
\hline \hline
\end{tabular}

collision cross sections for metal vapors are investigated. Then a method to calculate atomic absorption spectra is described. Next, DSMC results are compared with measured data. Finally, some conclusions are given.

\section{ATOMIC COLLISION CROSS SECTIONS FOR METAL VAPORS}

The inverse-power model is often employed to describe gaseous atomic or molecular interactions. ${ }^{2,6}$ According to the model, the force between the particles varies as some inverse power $\eta$ of the distance between their centers,

$$
F=\kappa / r^{\eta} \text {. }
$$

The constants $\kappa$ and $\eta$ can be adjusted to make the coefficient of viscosity conform to experimental data for a given gas over a range of conditions.

The collision cross section and coefficient of viscosity for the inverse-power model may be written as ${ }^{2,6}$

$$
\sigma_{T}=\pi W_{0, m}^{2}\left(\frac{\kappa}{m_{r} c_{r}^{2}}\right)^{2 /(\eta-1)},
$$

and

$$
\mu=\frac{\frac{5}{8} \sqrt{\pi m k T}}{(m / 4 k T)^{4} \int_{0}^{\infty} c_{r}^{7} \sigma_{\mu}\left(c_{r}\right) e^{-m c_{r}^{2} / 4 k T} d c_{r}},
$$

where $W_{0, m}$ is a constant, $m_{r}$ is the reduced mass, $c_{r}$ is the relative speed, $m$ is the atomic mass, $k$ is the Boltzmann constant, $T$ is the temperature, $\sigma_{\mu}$ is the viscosity cross section,

$$
\sigma_{\mu}=2 \pi\left(\frac{2 \kappa}{m}\right)^{2 /(\eta-1)} c_{r}^{-4 /(\eta-1)} A_{2}(\eta),
$$

and $A_{2}(\eta)$ is a numerical factor (see the Appendix). Substitution of Eq. (4) into Eq. (3) gives

$$
\mu=\frac{5(k m T / \pi)^{1 / 2}(2 k T / \kappa)^{2 /(\eta-1)}}{8 A_{2}(\eta) \Gamma[4-2 /(\eta-1)]} .
$$

In DSMC calculations, the variable hard sphere (VHS) model is often employed. ${ }^{2,13}$ The model assumes that the scattering during particle collisions is isotropic in the center of mass frame of reference, but the collision cross section has the same relation with the relative speed as the inversepower model,

$$
\sigma_{T}=\sigma_{T, \text { ref }}\left(c_{r, \text { ref }} / c_{r}\right)^{4 /(\eta-1)},
$$

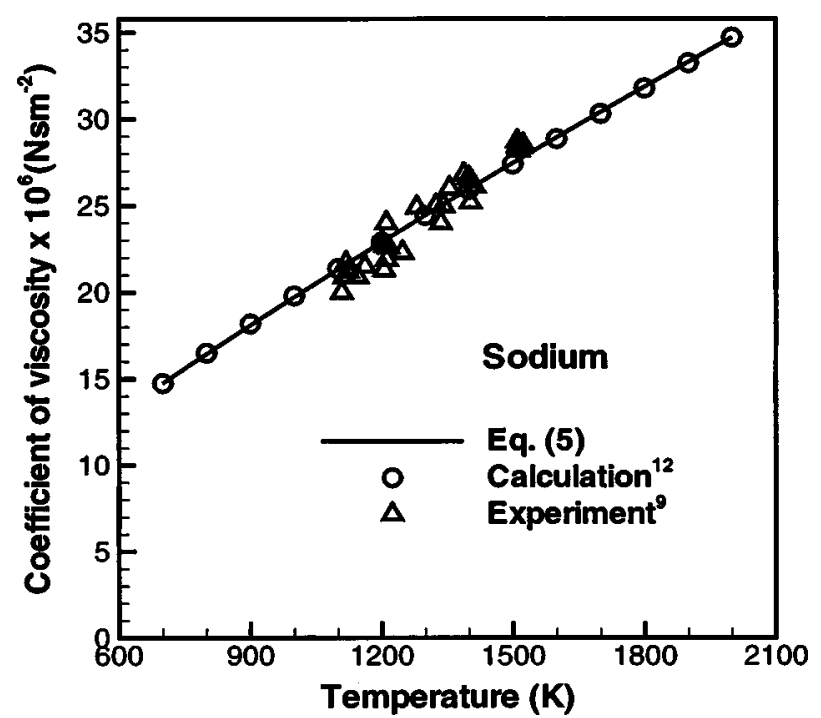

FIG. 1. Comparison of the coefficient of viscosity for sodium vapor vs temperature.

where subscript "ref', denotes the values at a reference temperature $T_{\text {ref }}$. It may further be shown that ${ }^{2,13}$

$$
\frac{\sigma_{T}}{\pi d_{\text {ref }}^{2}}=\frac{\left(2 k T_{\text {ref }} / m_{r} c_{r}^{2}\right)^{\omega-0.5}}{\Gamma(2-\omega)},
$$

with the reference collision diameter $\left(\pi d_{\text {ref }}^{2}=\sigma_{T, \text { ref }}\right)$,

$$
d_{\mathrm{ref}}=\left(\frac{15\left(m k T_{\mathrm{ref}} / \pi\right)^{0.5}}{2(5-2 \omega)(7-2 \omega) \mu_{\mathrm{ref}}}\right)^{0.5},
$$

where $\mu_{\text {ref }}$ is the coefficient of viscosity at $T_{\text {ref }}$, and $\omega$ $=1 / 2+(2 / \eta-1)$.

The values of $\eta$ and $\kappa$ for alkali metal vapors have been obtained by equating the right-hand side of Eq. (5) with theoretical data $^{12}$ at temperatures of 700 and $2000 \mathrm{~K}$, respectively, and these are given in Table I. Figures 1 and 2 com-

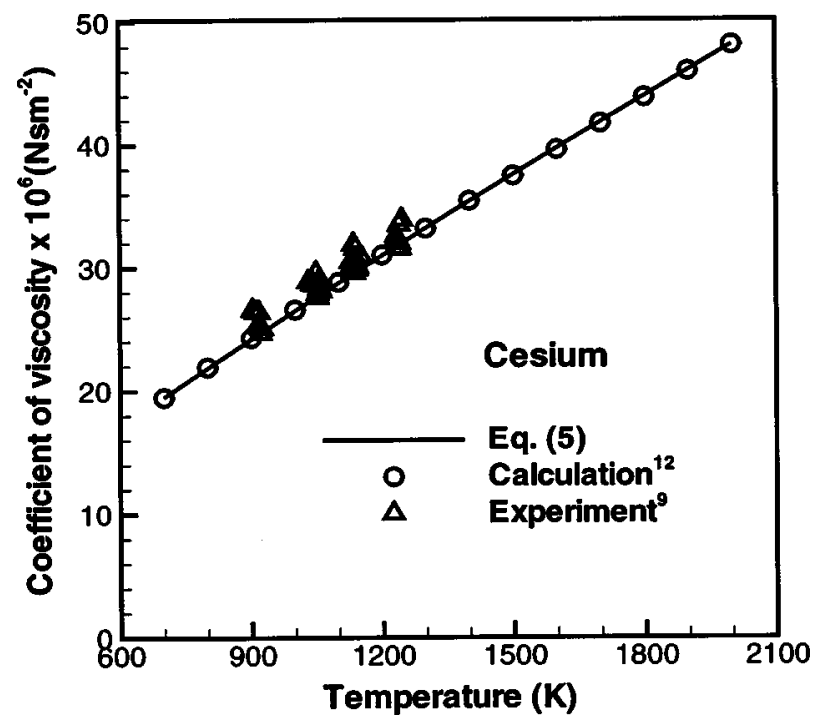

FIG. 2. Comparison of the coefficient of viscosity for cesium vapor vs temperature. 
TABLE II. Parameters of the VHS model for metal vapors at $T_{\text {ref }}=2000 \mathrm{~K}$

\begin{tabular}{ccc}
\hline \hline Element & $d_{\text {ref }} \times 10^{10} \mathrm{~m}$ & $\omega$ \\
\hline Lithium & 4.562 & 0.875 \\
Sodium & 4.961 & 0.815 \\
Potassium & 5.554 & 0.849 \\
Rubidium & 5.846 & 0.853 \\
Cesium & 6.306 & 0.862 \\
Barium & 6.358 & 0.862 \\
Yttrium & 5.903 & 0.853 \\
Zirconium & 5.941 & 0.853 \\
Copper & 6.271 & 0.849 \\
Titanium & 5.844 & 0.849 \\
\hline \hline
\end{tabular}

pare Eq. (5) to the theoretical and experimental data for sodium and cesium. The profiles of Eq. (5) are in excellent agreement with the theoretical results; ${ }^{12}$ the experimental data, ${ }^{9}$ although with scatter, support the theoretical results and fitted profiles in their trend.

The reference collision diameters of the VHS model ${ }^{2}$ for the alkali metal vapors have been obtained using Eqs. (5) and (8), and are given in Table II. We assume that the values of $\eta$ and $\kappa$ for the alkali metal vapors apply to other metal vapors occupying the same row in the periodic table of elements. The values of $d_{\text {ref }}$ for several metal vapors of interest based on this assumption are calculated and given in Table II. The selection of the reference temperature is arbitrary and does not affect collision cross sections. To be reasonable physically, it is taken to be $2000 \mathrm{~K}$ since that is above the melting point of all the elements considered in Table II.

Atomic collision cross sections of metal vapors are much larger in comparison with common atomic gases. Figure 3 compares the VHS collision cross section for sodium vapor to that for argon gas with $d_{\text {ref }}=4.17 \times 10^{-10} \mathrm{~m}$ at $T_{\text {ref }}$ $=273 \mathrm{~K}$ (Table A2 in Ref. 2). The sodium profile is three to four times higher than the argon profile. This is mainly due

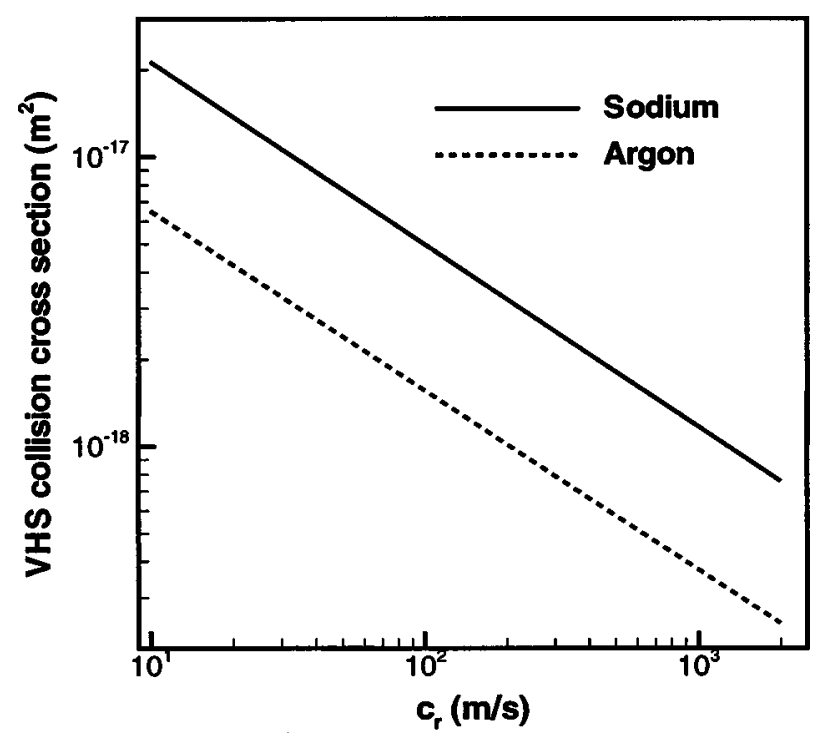

FIG. 3. Comparison of the VHS collision cross sections for sodium vapor and argon gas vs relative speed. to the larger viscosity of argon which, for example, is $5.452 \times 10^{-5} \mathrm{~N} \mathrm{~m} \mathrm{~s}^{-2}$ at $700 \mathrm{~K}$ and $0.1 \mathrm{MPa},{ }^{14}$ while the viscosity of sodium vapor at the same conditions is 1.475 $\times 10^{-5} \mathrm{~N} \mathrm{~m} \mathrm{~s}^{-2}{ }^{12}$ The larger collision cross sections of the metal vapors result in more frequent collisions between the atoms and have an important effect on the gas dynamics of the plume expansion, thus affecting the film growth rate and area of uniform deposition. These aspects will be considered in Sec. IV.

\section{ATOMIC ABSORPTION SPECTRA}

In situ monitoring and control is one of the primary issues involved in EBPVD processing of superconducting film. ${ }^{15}$ Diode-laser-based atomic absorption monitors for yttrium and barium have been developed to meet this need. ${ }^{16}$ They are simultaneously both element specific and noninvasive, and can provide the atomic absorption spectrum from which the atomic flux may be derived.

Using the monitors, an atomic absorption spectral distribution around the central frequency $v_{0}$ along a laser path may be observed. The spectrum $I(v)$ in the vicinity of $v_{0}$ is called the line profile. The profile may have many peaks centered at $v_{i}\left(v_{i}=v_{0}+\Delta v_{i}\right.$, and $\left.\Delta v_{i} \ll v_{0}\right)$, respectively, that correspond to the frequencies absorbed by the atoms/ molecules when the light beam traverses the subject material. These peaks usually have a finite width due to various effects of broadening. In low pressure $\left(<10^{-2}\right.$ Torr) situations, the width is mainly determined by the Doppler effect. ${ }^{17}$ This takes place because the frequency absorbed by a particle with a velocity component in the direction of the light beam is slightly different from that absorbed by a stationary particle. At thermal equilibrium, a Doppler-broadened peak shape centered at the frequency $v_{i}$ is described by ${ }^{17}$

$$
I_{i}(v)=\frac{\beta \lambda_{i}}{\sqrt{\pi}} \exp \left[-\beta^{2} \lambda_{i}^{2}\left(v-v_{i}\right)^{2}\right]
$$

where the wavelength $\lambda_{i}=c / v_{i}, c$ is the light speed, and $\beta$ $=\sqrt{m / 2 k T}$.

The spectral distribution, Eq. (9), corresponds to a Maxwellian velocity distribution,

$$
f\left(\omega_{l}\right)=\frac{\beta}{\sqrt{\pi}} \exp \left(-\beta^{2} \omega^{2}\right),
$$

where $\%$ is the thermal velocity component in the light-beam direction, which causes the Doppler frequency shift. Comparison of the two distributions, Eqs. (9) and (10), leads to the relations,

$$
v-v_{i}=v_{0} / \lambda_{i},
$$

and

$$
I_{i}(v)=\lambda_{i} f\left(v_{\ell}\right) .
$$

Equations (11) and (12) provide a means by which to convert a velocity distribution of particles into an absorption scan. There are two ways to calculate the velocity distribution using the DSMC method. One is through direct sam- 
pling of the particle velocities along the laser path; the other is based on the assumption of Maxwellian velocity distributions characterized by the temperature, velocity, and density in the cells along the laser path. Note that the latter approach is numerically less expensive. Results from both of these approaches will be provided. The calculated velocity distribution function must be normalized in accordance with the experimental spectrum $I^{\exp }(v)$. The normalization condition requires

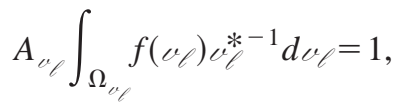

and

$$
B_{v} \int_{\Omega_{v}} I_{i}^{\exp }(v) v^{*-1} d v=1,
$$

where $u^{*}$ and $v^{*}$ are velocity and frequency normalization factors, respectively, and $A_{v}$ and $B_{v}$ are overall normalization factors. Relation (11) requires

$$
v^{*}=v^{*} / \lambda_{i}
$$

The hyperfine electronic structure of absorption spectra is another issue that must be considered. ${ }^{18}$ For example, a line profile for the ground state transition of yttrium at $v_{0}$ $=4.486 \times 10^{14} \mathrm{~Hz}$ is the sum of four components with $\Delta v_{i}$ and relative intensity factors $\gamma_{i}$ given in Table III. Thus the calculated line profile $I^{\mathrm{cal}}(v)$ is the weighted sum of the four peak shapes centered at slightly different frequencies $v_{i}$ $=v_{0}+\Delta v_{i}$,

$$
I^{\mathrm{cal}}(v)=\sum_{i=1}^{4} \gamma_{i} I_{i}^{\mathrm{cal}}(v) / \sum_{i=1}^{4} \gamma_{i}
$$

\section{EXPERIMENTAL AND COMPUTATIONAL CONDITIONS}

The top and side views of the experimental deposition chamber are shown in Fig. 4. The yttrium, barium, and copper pool surfaces are designed to be oval and of the same size. We consider two cases where only the yttrium source is evaporating. The evaporation rates are measured by the feed rate of the ingot into the chamber, and are given in Table III. The pool surface temperatures are calculated from ${ }^{19}$

$$
\log _{10} W=C-0.5 \log _{10} T-B / T,
$$

where $W$ denotes the evaporation rate in units of $\mathrm{g} / \mathrm{cm}^{2} \mathrm{~s}$, and $B$ and $C$ are constants that are $2.197 \times 10^{4}$ and 9.17 , respectively, for yttrium (cf. Table 10.2 in Ref. 19).

DSMC calculations are carried out using a software system called MONACO-YBC. ${ }^{20}$ The system is developed from the original MONACO code ${ }^{3,4}$ that employed a localized data structure based on a computational cell to achieve high performance on workstation processors. Besides the translational energy mode, the atomic electronic energy is taken into account using the model described in Ref. 5. About two

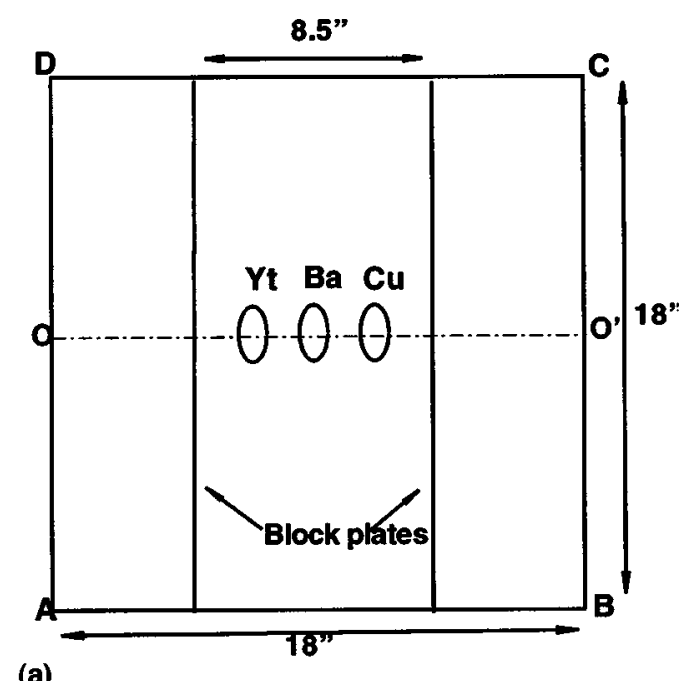

(a)

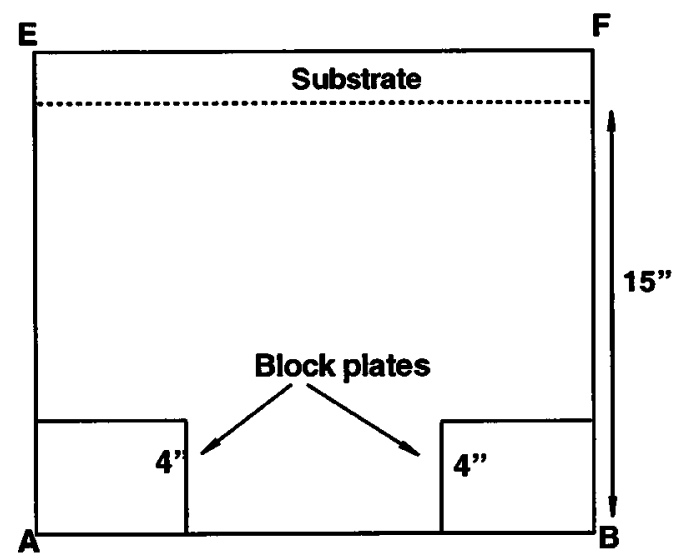

(b)

FIG. 4. Schematic diagram of the deposition chamber.

million simulated particles are used in the calculations. The results are obtained overnight on a SGI OCTANE workstation.

An orthogonal coordinate system with the origin $O$ is employed: the $x$ and $y$ axes are along $O O^{\prime}$ and $O A$, respectively, and the $z$ axis points to the substrate (cf. Fig. 4). The computational grid employs $20 \times 60 \times 50$ cuboid cells. The cell sizes are nonuniform: a very small cell size in the $z$ direction is employed to capture the rapid jet expansion near the evaporation source, and small cell sizes in the $x$ and $y$ directions are used to resolve the flow in the region covering the source that is most important for accurate simulation of the deposition process.

TABLE III. Hyperfine structure parameters of atomic absorption spectra for yttrium.

\begin{tabular}{ccc}
\hline \hline & $\begin{array}{c}\Gamma_{e} \\
(\mathrm{~mole} / \mathrm{s})\end{array}$ & $\begin{array}{c}T_{s} \\
\text { Case }\end{array}$ \\
\hline I & $9.95 \times 10^{-5}$ & 2091 \\
II & $8.42 \times 10^{-6}$ & 1881 \\
\hline \hline
\end{tabular}




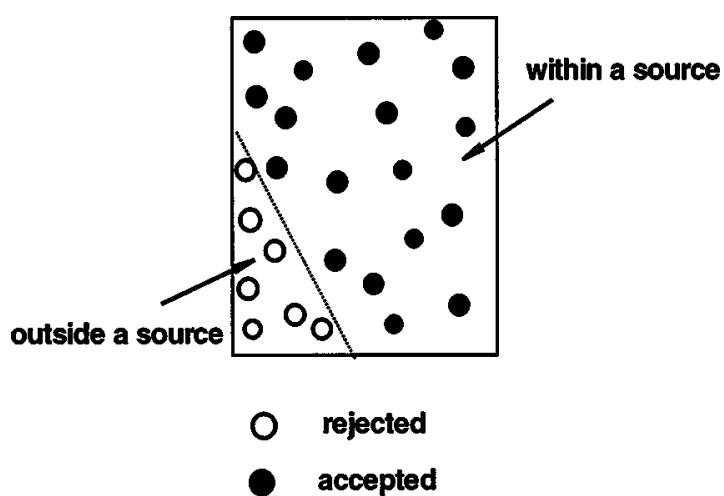

FIG. 5. Determination of inflow atoms.

The chamber walls and block plate surfaces are assumed to be perfectly sticking. This assumption is well satisfied because the wall and surface temperatures are low in this study. New particles are vaporized into the chamber from the oval surface of the yttrium pool each time step. The pool surface is described by a certain number of surface elements. If a surface element is wholly within the source, all particles generated at the known evaporation rate will be permitted to enter the chamber. Because the cells are cuboid and the pool surface is oval, a surface element may lie only partly within the source (Fig. 5). In this situation, only the particles generated in a region within the source are actually accepted; the particles generated in a region outside it are rejected.

When particles evaporate from the surface into the chamber, they may collide with one another immediately above the pool, particularly for cases with high evaporation rates. This causes a fraction of the atoms to develop postcollision velocities that move them back to the molten pool. In Ref. 5, the total backscatter flux was found to be as high as $19 \%$ of the evaporating rate. There are two ways to maintain a net inflow rate measured by the feed rate of the ingot into the chamber. One is based on iteration, i.e., correcting the inflow flux using the backscatter fraction until the net inflow rate is the same as the measured data, ${ }^{5}$ another is to let the backscattered particles diffusively reflect from the pool surface. The latter approach is numerically inexpensive and is employed here.

\section{RESULTS AND DISCUSSION}

\section{A. Case I: High evaporation rate}

Figures 6(a)-6(c) show the computed number density, translational temperature, and velocity fields. A rapid expansion occurs around the source. Within a distance of about $0.05 \mathrm{~m}$, the velocity in the $z$ direction increases from about 100 to $700 \mathrm{~m} / \mathrm{s}$, while the number density and translational temperature decrease from $10^{20} \mathrm{~m}^{-3}$ and $1500 \mathrm{~K}$ to $10^{18} \mathrm{~m}^{-3}$ and $250 \mathrm{~K}$, respectively. Thereafter, variation of all flow properties is relatively slow.

Figure 7 shows variation of two continuum breakdown parameters along a path from the yttrium source center in the direction perpendicular to the substrate. One was proposed by Bird for expanding flows, ${ }^{21}$

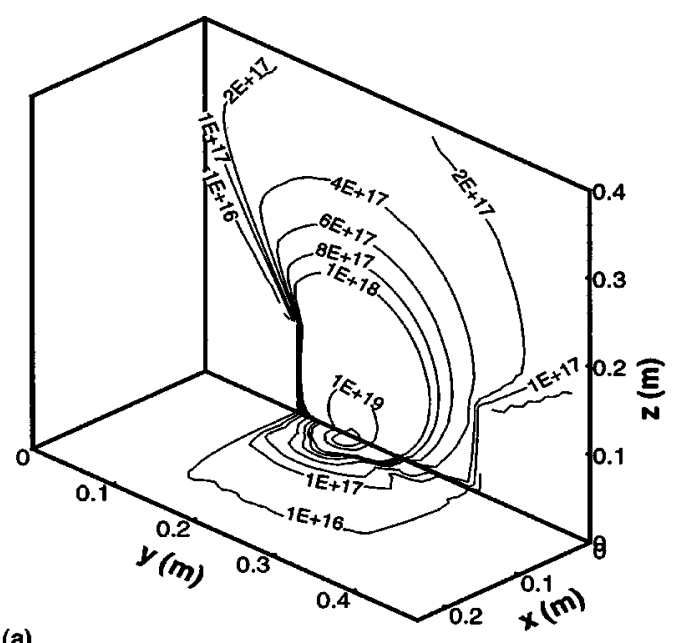

(a)

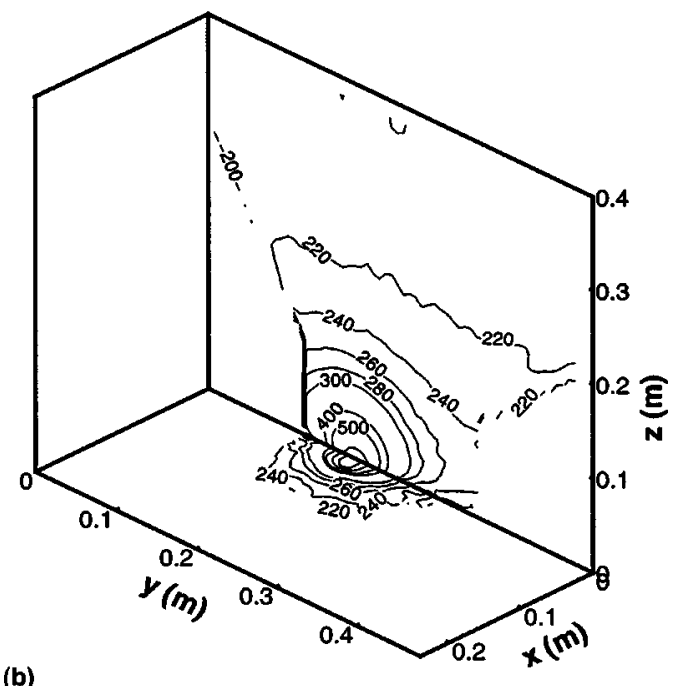

(b)

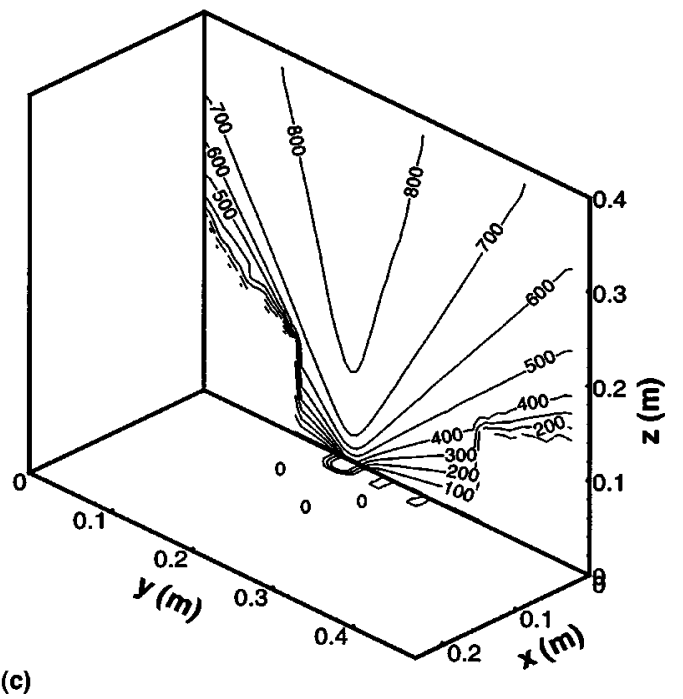

FIG. 6. Flow field given by the DSMC method for case I. (a) number density $\left(\mathrm{m}^{3}\right)$; (b) translational temperature $(\mathrm{K})$; (c) velocity in the $z$ direction.

$$
P=\frac{U}{\rho v}\left|\frac{d \rho}{d s}\right|=M \sqrt{\frac{\pi \gamma}{8}} \frac{\lambda}{\rho}\left|\frac{d \rho}{d s}\right|,
$$

where $U$ is the local velocity, $v$ is the local collision fre- 


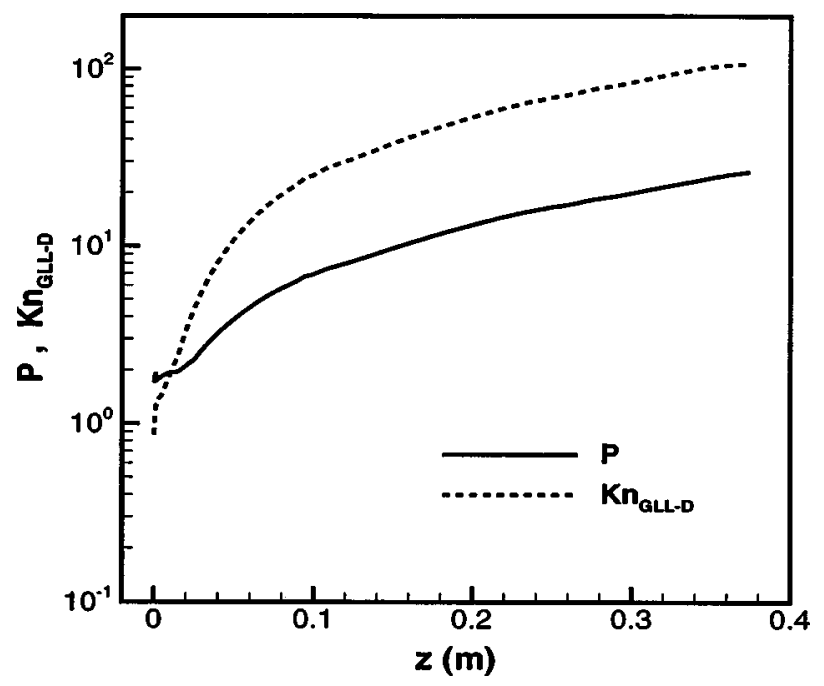

FIG. 7. Variation of the Knudsen number along a path from the yttrium source center perpendicular to the substrate for case I.

quency, $M$ is the local Mach number, $\gamma$ is the specific heat ratio, and $s$ is the distance along a streamline. Studies in Ref. 21 indicate that the continuum approach fails in expanding flows at a value for $P$ of about 0.05 . The other breakdown parameter was proposed by Boyd, Chen, and Candler. ${ }^{22}$ Comparison between DSMC and Navier-Stokes results for shock waves and hypersonic flows around a sphere showed that the gradient-length local (GLL) Knudsen number based on density,

$$
\mathrm{Kn}_{\mathrm{GLL}-D}=\frac{\lambda|\nabla \rho|}{\rho},
$$

can more precisely specify the limit at which the NavierStokes equations are invalid. The continuum approach broke down wherever the value of $\mathrm{Kn}_{\mathrm{GLL}-D}$ exceeded 0.05. ${ }^{22}$ Both parameters show that the flow is completely in the noncontinuum regime, and needs to be studied based on the kinetic point of view.

Figure 8 shows a comparison of the DSMC deposition thickness profile along the symmetric line of the substrate with measured data. The collisionless result computed using DSMC but with atomic collisions turned off is also shown. The experimental data are obtained over a deposition time of $12 \mathrm{~min}$. The thickness is measured with a profilometer across a film edge which is created by blocking the substrate with adhesive tape. The substrate used is a polished silicon wafer. Since the coating is rather thick and the blistering of the film does not allow accurate sampling points, scatter in the measured data is clearly seen. The comparison between the calculated and measured results is reasonable. The collisionless profile is significantly lower than the DSMC profile and the measured data. The atomic collisions impede to some extent the atomic diffusion to the block plates and chamber walls that are assumed to be perfectly sticking. Neglecting the collisions results in the atoms having more chance to stick on the block plate surfaces or chamber walls rather than be deposited on the substrate. Figure 9 shows the computed depo-

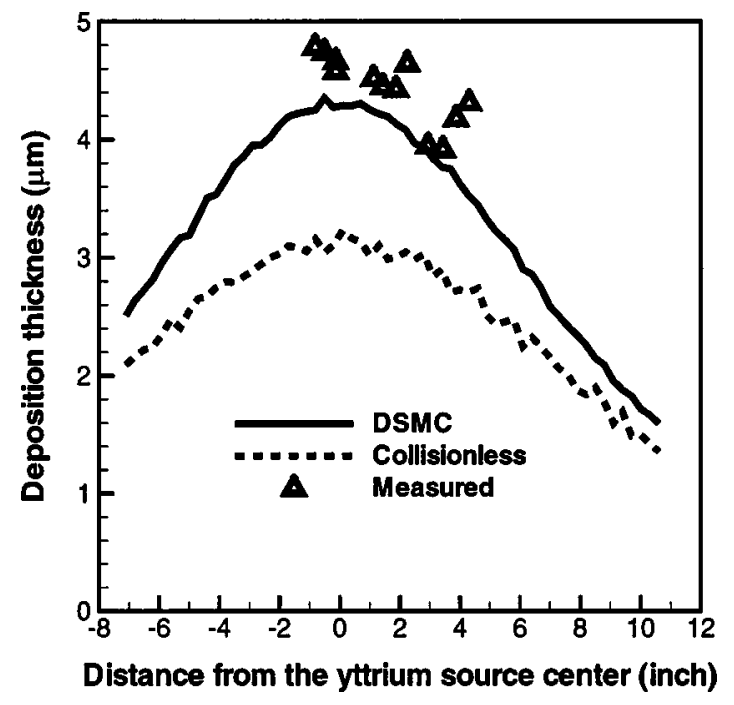

FIG. 8. Comparison of DSMC and experimental deposition thickness profiles for case I.

sition thickness distribution over the whole substrate. There is a region with a thickness of around $4.2 \mu \mathrm{m}$, whose diameter is about $0.8 \mathrm{in}$. and its center corresponds to the yttrium source center. The thickness decreases as the distance from the core increases. The value along the edges is about one third that of the core value.

Figure 10 gives a comparison of DSMC and measured atomic absorption spectra at frequencies of $4.486 \times 10^{14}$ and $4.416 \times 10^{14} \mathrm{~Hz}$ along an aperture close to the symmetric line of the substrate. The calculated and measured Doppler widths and peak-structure details are all in excellent agreement. Because of different hyperfine electronic structure at the two frequencies (cf. Table IV), the corresponding peak structures are inclined to the right and to the left, respectively.

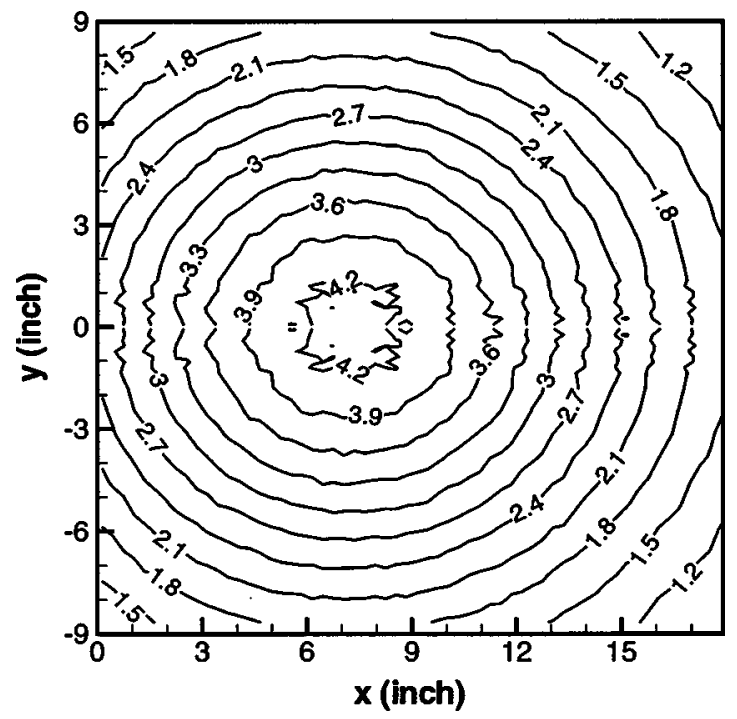

FIG. 9. Deposition thickness distribution over the whole substrate for case I. 


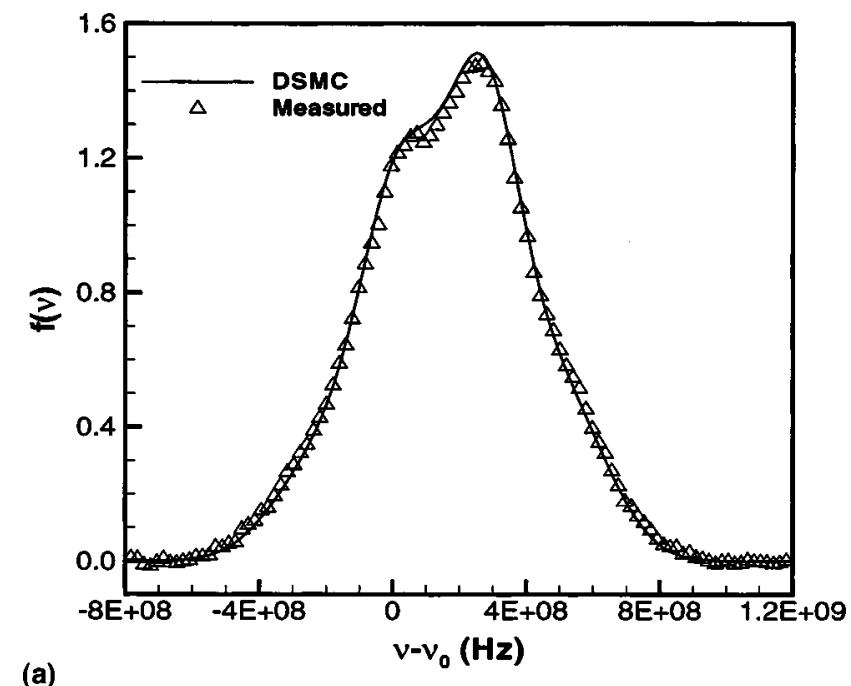

(a)

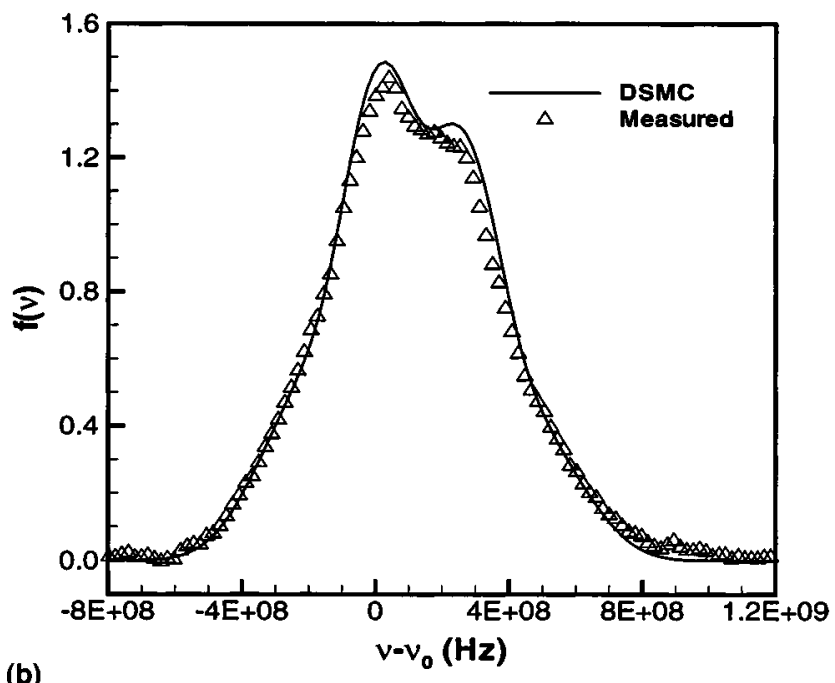

(b)

FIG. 10. Comparison of DSMC and experimental atomic absorption spectra for case I: (a) $v_{0}=4.486 \times 10^{14}$ and (b) $4.416 \times 10^{14} \mathrm{~Hz}$.

\section{B. Case II: Low evaporation rate}

Figure 11 shows the computed computer number density. Since the present evaporation rate is about one tenth that of case I, the largest number density around the source decreases to about $10^{19} \mathrm{~m}^{-3}$, that is, one tenth that for case I, whereas the evolutions along the $z$ direction exhibited by Figs. 11 and 6(a) are similar.

TABLE IV. Hyperfine structure parameters of atomic absorption spectra for yttrium.

\begin{tabular}{rlc}
\hline \hline$v_{0} \times 10^{14}(\mathrm{~Hz})$ & 4.486 & 4.416 \\
$\Delta v_{1} \times 10^{8}(\mathrm{~Hz})$ & 0.00 & 0.00 \\
$\Delta v_{2} \times 10^{8}(\mathrm{~Hz})$ & 1.14 & 0.86 \\
$\Delta v_{3} \times 10^{8}(\mathrm{~Hz})$ & 1.76 & 1.99 \\
$\Delta v_{4} \times 10^{8}(\mathrm{~Hz})$ & 2.90 & 2.85 \\
$\gamma_{1}$ & 5 & 20 \\
$\gamma_{2}$ & 1 & 1 \\
$\gamma_{3}$ & 1 & 1 \\
$\gamma_{4}$ & 9 & 14 \\
\hline \hline
\end{tabular}

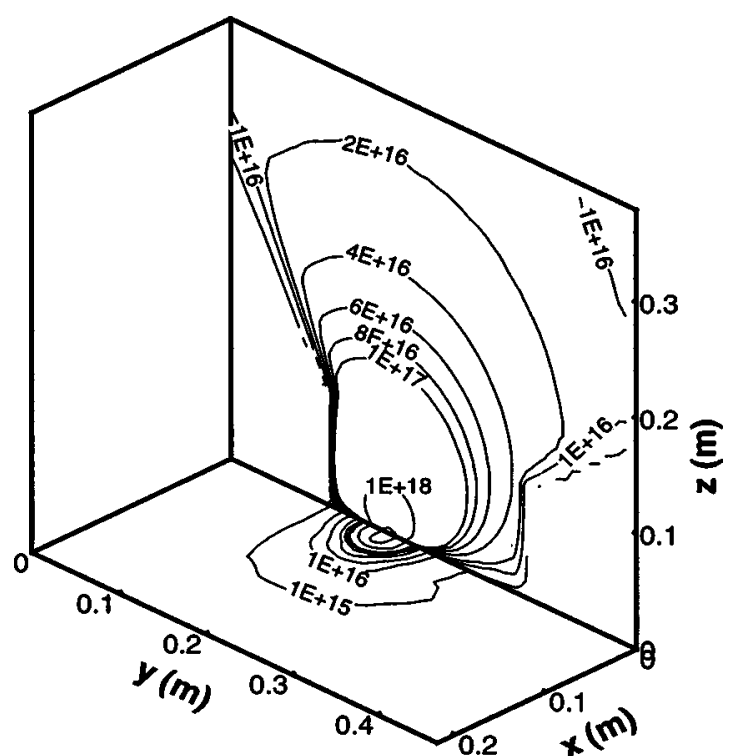

FIG. 11. DSMC number density field $\left(\mathrm{m}^{3}\right)$ for case II.

The continuum breakdown parameters Eqs. (18) and (19), are computed along a path from the yttrium source center perpendicular to the substrate and are shown in Fig. 12. The parameter $P$ is greater than 10 except in a region close to the source. This means that the flow is close to the free molecular limit.

Figure 13 is a comparison of the DSMC deposition thickness profile with measured data and the collisionless result. In the experiment, the deposition time is $30 \mathrm{~min}$. A quartz crystal monitor was used to measure the atomic flux rate toward the substrate. The rate integration provided the thickness data that were verified by the profilometer measurement. The DSMC profile agrees quite well with the measured data. The agreement between the DSMC and collisionless profiles is in accordance with the prediction given by the continuum breakdown parameters.

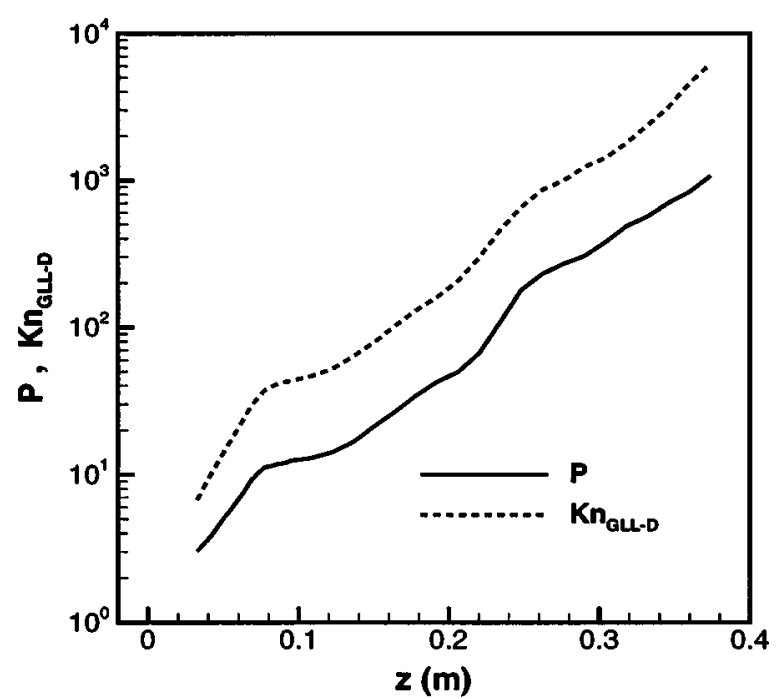

FIG. 12. Variation of the Knudsen number along a path from the yttrium source center perpendicular to the substrate for case II. 


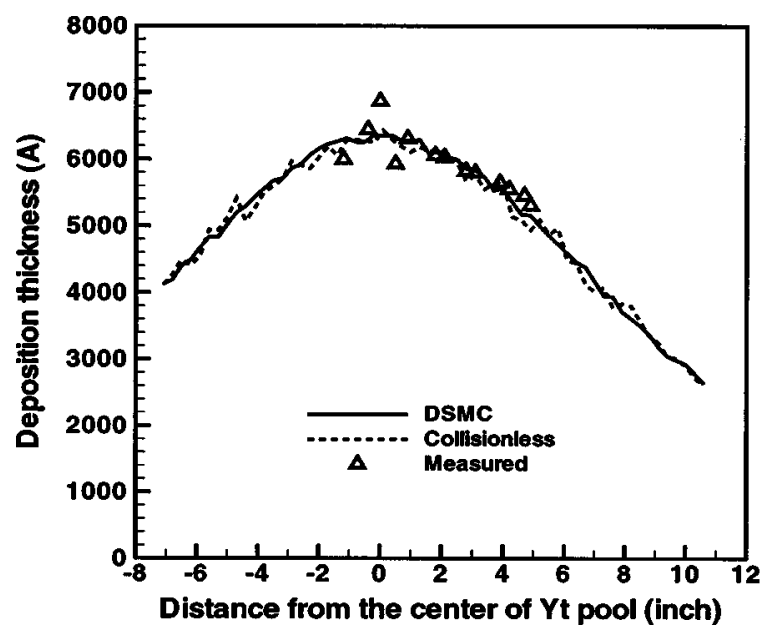

FIG. 13. Comparison of DSMC and experimental deposition thickness profiles for case II.

DSMC and measured atomic absorption spectra centered at a frequency of $4.486 \times 10^{14} \mathrm{~Hz}$ are compared in Fig. 14 . They agree well with each other in their trend. Because of the decrease of number density, the light intensity variation caused by the atomic absorption is small, i.e., the measured signal accepted by the spectrometer is weak. This results in significant scatter in the measured data.

\section{CONCLUSIONS}

A three-dimensional yttrium vapor deposition process was investigated using the DSMC method and experiment. The atomic absorption spectra and deposited film thickness profiles of the yttrium vapor plumes given by the DSMC method and experiment at the two different evaporation rates were both in excellent agreement.

Atomic collision cross sections for metal vapors were studied. The parameters of collision cross sections for the variable hard-sphere model ${ }^{2,13}$ were obtained for alkali metal vapors and other metal vapors of interest such as yttrium,

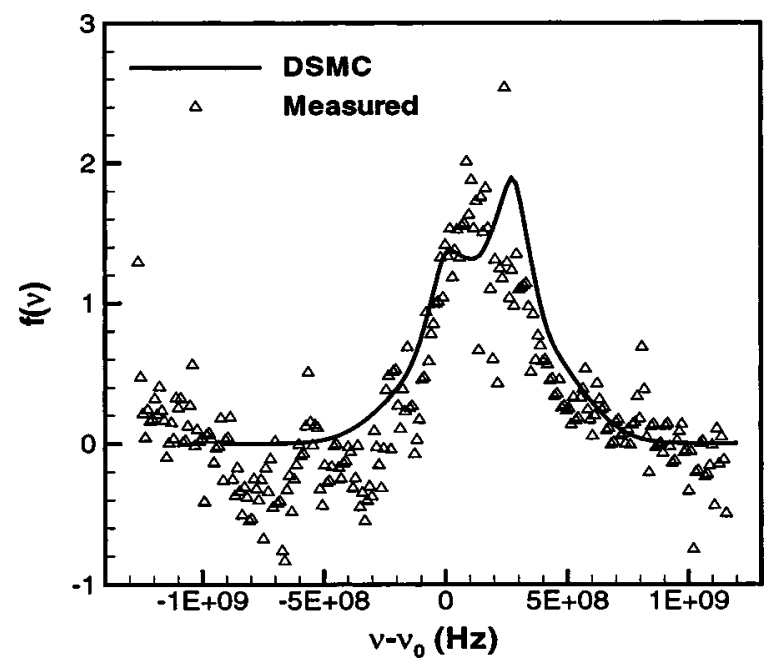

FIG. 14. Comparison of DSMC and experimental atomic absorption spectra with $v_{0}=4.486 \times 10^{14} \mathrm{~Hz}$ for case II. barium, and copper. The values for yttrium vapor were used in the DSMC calculations. The excellent agreement of the DSMC and experimental results indicated that the approach used to determine the collision cross sections for metal vapors was reasonable.

Collisions between the yttrium atoms were found to have an important effect on the film growth rate and the area of uniform deposition as the evaporation rate increased. Collisions determine the atomic trajectories and whether particles move toward the substrate or toward the chamber walls or block plates that were assumed to be perfectly sticking. For the case with the high evaporation rate, the DSMC calculations showed that the atomic collisions may result in a significant increase of the deposition rate and a decrease of deposition uniformity.

The hyperfine electronic structure significantly affected the atomic absorption spectra of yttrium. For instance, in the case with the high evaporation rate, because of the different hyperfine structure at $v_{0}=4.486 \times 10^{14}$ and $4.416 \times 10^{14} \mathrm{~Hz}$, the DSMC and experimental atomic absorption peaks at the two frequencies were inclined to the left and to the right, respectively.

\section{ACKNOWLEDGMENTS}

This work was developed as part of the Office of Naval Research/3M "Models, Sensors, and Controls for E-Beam Deposition" program, Agreement No. N00014-98-3-0015. The content does not necessarily reflect the position or policy of the government and no official endorsement should be inferred.

\section{APPENDIX: CALCULATION OF THE VISCOSITY CROSS-SECTION COEFFICIENT}

The viscosity cross-section coefficient $A_{2}(\eta)$ is defined $\operatorname{as}^{2,6}$

$$
A_{2}(\eta) \equiv \int_{0}^{\infty} \sin ^{2} \chi \alpha d \alpha,
$$

with

$$
\chi=\pi-2 \int_{0}^{w_{+}}\left[1-x^{2}-\frac{2}{\eta-1}\left(\frac{x}{\alpha}\right)^{\eta-1}\right]^{-(1 / 2)}
$$

where $w_{+}$is the (unique) positive root of

$$
1-w^{2}-\frac{2}{\eta-1}\left(\frac{w}{\alpha}\right)^{\eta-1}=0 .
$$

Table 3 in Ref. 6 tabulated the values of $A_{2}(\eta)$ for the cases of $\eta=5,7,9,11,15,21,25$, and $\infty$ that were contributed by many researchers with the original literature given in a note in Ref. 6 (p. 172). For cases in which $\eta$ is not an integer, Eqs. (A1)-(A3) may be solved numerically. Equation (A3) is transformed into

$$
w=\alpha\left(\frac{(\eta-1)\left(1-w^{2}\right)}{2}\right)^{(1 / \eta-1)} .
$$




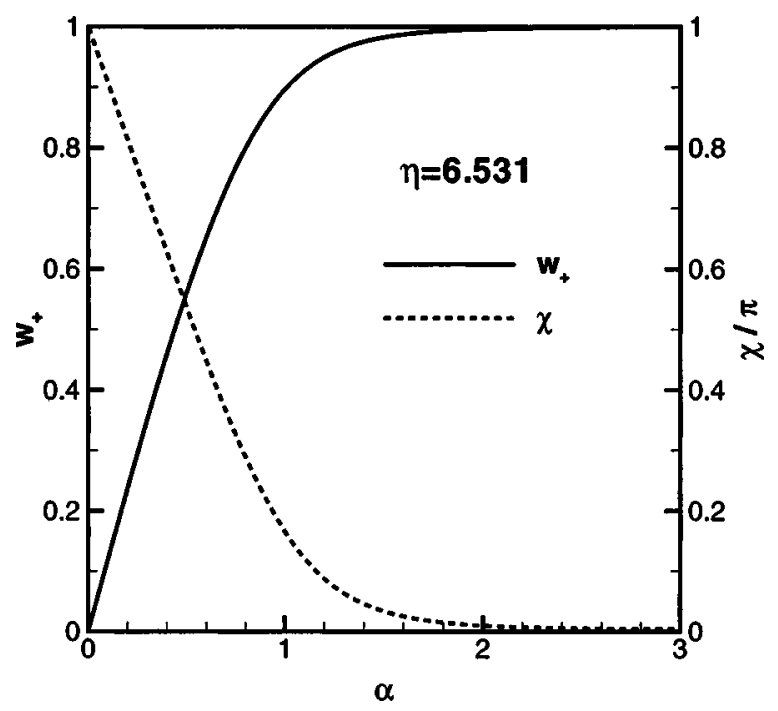

FIG. 15. Relation of $w_{+}$and $\chi$ vs $\alpha$ for $\eta=6.531$.

It may be solved through a numerical relaxation iteration. Then the corresponding values of $\chi$ and $A_{2}(\eta)$ themselves are obtained from Eqs. (A2) and (A1), respectively, by numerical integration. The relation of $w_{+}$and $\chi$ vs $\alpha$ for $\eta$ $=6.531$, which is the value for cesium vapor, is shown in Fig. 15.

${ }^{1}$ C. C. Chi and R. B. Dover, High $T_{c}$ Superconducting Thin Films and Applications (SPIE, Bellingham, WA, 1990).

${ }^{2}$ G. A. Bird, Molecular Gas Dynamics and the Direct Simulation of Gas Flows (Clarendon, Oxford, 1994).

${ }^{3}$ S. Dietrich and I. D. Boyd, J. Comput. Phys. 126, 328 (1994).

${ }^{4}$ K. C. Kannenberg and I. D. Boyd, J. Thermophys. Heat Transfer 13, 226 (1999).
${ }^{5}$ J. Balakrishnan, I. D. Boyd, and D. G. Braun, J. Vac. Sci. Technol. A 18, 907 (2000)

${ }^{6} \mathrm{~S}$. Chapman and T. G. Cowling, The Mathematical Theory of Nonuniform Gases (Cambridge University Press, London, 1952).

${ }^{7}$ W. A. Wakeham, A. Nagashima, and J. V. Sengers, Measurement of the Transport Properties of Fluids (IUPAC/Blackwell Scientific, London, 1991), Vol. III.

${ }^{8}$ N. B. Vargaftik and V. S. Yargin, Handbook of Thermodynamic and Transport Properties of Alkali Metals, edited by R. W. Ohse (Blackwell Scientific, London, 1985), pp. 785-842.

${ }^{9}$ N. B. Vargaftik, V. S. Vinogradov, V. I. Dolgov, V. G. Dzis, I. F. Stepanenko, Y. K. Yakimovich, and V. S. Yargin, Int. J. Thermophys. 12, 85 (1991).

${ }^{10}$ P. S. Fialho, M. L. V. Ramires, C. A. Nieto de Castro, J. M. N. A. Fareleira, and U. V. Mardolcar, Ber. Bunsenges. Phys. Chem. 98, 92 (1994).

${ }^{11}$ C. A. Nietro de Castro, J. M. N. A. Fareleira, P. M. Matias, M. L. V. Ramires, A. A. C. Canelas, and A. J. C. Varandas, Ber. Bunsenges. Phys. Chem. 94, 53 (1990).

${ }^{12}$ P. S. Fialho, M. L. V. Ramires, J. M. N. A. Fareleira, and C. A. Nietro de Castro, Ber. Bunsenges. Phys. Chem. 97, 1487 (1993).

${ }^{13}$ G. A. Bird, Prog. Astronaut. Aeronaut. 74, 239 (1981).

${ }^{14}$ J. Kestin and W. A. Wakeham, Transport Properties of Fluids: Thermal Conductivity, Viscosity, and Diffusion Coefficient (Hemisphere, New York, 1988).

${ }^{15}$ M. S. DiIorio, in High $T_{c}$ Superconducting Thin Films and Applications, edited by C. C. Chi and R. B. Dover (SPIE, Bellingham, WA, 1990), pp. $2-15$.

${ }^{16}$ W. Wang, R. H. Hammond, M. M. Fejer, and M. R. Beasley, J. Vac. Sci. Technol. A 17, 2676 (1999).

${ }^{17}$ W. Demtroder, Laser Spectroscopy: Basic Concepts and Instrumentation, 2nd enlarged ed. (Springer, New York, 1996).

${ }^{18}$ W. Wang, R. H. Hammond, M. M. Fejer, S. Arnason, and M. R. Beasley, Appl. Phys. Lett. 71, 31 (1997).

${ }^{19}$ S. Dushman, Scientific Foundation of Vacuum Technique (Wiley, New York, 1962)

${ }^{20}$ J. Fan and I. D. Boyd, The MONACO-YBC System for Simulating E-Beam Deposition, Release Ver. 1.0, The University of Michigan, Ann Arbor, January 2000.

${ }^{21}$ G. A. Bird, AIAA J. 8, 1998 (1970).

${ }^{22}$ I. D. Boyd, G. Chen, and G. V. Candler, Phys. Fluids 7, 210 (1995). 